\title{
Le système éducatif vietnamien
}

\section{Sébastien Asseraf-Godrie}

\section{(2) OpenEdition}

Journals

Édition électronique

URL : https://journals.openedition.org/ries/789

DOI : $10.4000 /$ ries.789

ISSN : 2261-4265

\section{Éditeur}

France Education international

\section{Édition imprimée}

Date de publication : 1 décembre 2009

Pagination : 148-154

ISBN : 978-2-8542-0577-0

ISSN : 1254-4590

\section{Référence électronique}

Sébastien Asseraf-Godrie, "Le système éducatif vietnamien ", Revue internationale d'éducation de Sèvres [En ligne], 52 I décembre 2009, mis en ligne le 01 décembre 2011, consulté le 26 avril 2022 URL : http://journals.openedition.org/ries/789; DOI : https://doi.org/10.4000/ries.789

Ce document a été généré automatiquement le 26 avril 2022

(c) Tous droits réservés 


\title{
Le système éducatif vietnamien
}

\author{
Sébastien Asseraf-Godrie
}

1 Avec un taux de croissance du PIB entre 6 et 8,5\% depuis plus de dix ans, la République socialiste du Viêt Nam a besoin d'une main-d'œuvre qualifiée importante pour pérenniser son développement. La demande sociale d'éducation est forte. Il s'agit d'y répondre en assurant à la fois un accès équitable, une bonne qualité des acquis des élèves et étudiants, le tout en adéquation avec le développement du pays et la modernisation en cours. C'est dans ce contexte qu'une grande réforme du système éducatif vietnamien est en cours d'élaboration : un nouveau plan stratégique s'attaque à la fois à l'organisation et au financement de l'éducation mais aussi, à sa conception et à ses héritages culturels.

\section{Contexte historique ${ }^{1}$}

De - 111 à 939, le Viêt Nam est annexé à la Chine des Hán. Pendant 1500 ans la langue hán, le chinois classique, y est langue de communication, comme dans toute l'Asie orientale. Les conséquences de cette domination restent visibles dans la culture, certains comportements et le vocabulaire. L'éducation est alors celle des enfants hán, pour l'essentiel les préparant au fonctionnariat. Les tentatives de réforme, au XIX ${ }^{\mathrm{e}}$ siècle, d'un système figé par la tradition échouent devant le conservatisme étroit de la monarchie ${ }^{2}$. Les concours d'entrée à la fonction publique calqués sur le modèle confucéen ne disparaissent qu'en 1917, au moment de l'ouverture de l'université.

3 À la seconde moitié du XIX ${ }^{\mathrm{e}}$ siècle, les Français tentent d'imposer au Protectorat l'apprentissage du français dès le primaire afin, à leur tour, de former des cadres. Dans le même temps, après des hésitations politiques, l'administration française favorise la diffusion de l'écriture vietnamienne romanisée, le quôc-ngu ${ }^{3}$, adoptée par les élites vietnamiennes (souvent catholiques), par les écoles traditionnelles et par la presse nationaliste de la bourgeoisie naissante. Mais l'école "franco-indigène », où l'on enseigne la langue vie.t et le français, reste très sélective et une grande partie de la population est encore analphabète. En 1943-1944, 3,2 \% de la population est scolarisée. 
4 À la suite de l'indépendance proclamée le 2 septembre 1945, le gouvernement de la République démocratique du Viêt Nam se lance dans une campagne d'alphabétisation liée à une campagne de propagande intense. Le 8 septembre est créé le Département de l'éducation populaire qui organise une formation massive et accélérée d'alphabétiseurs. À la fin des années 50, à l'exception de celle des hauts plateaux, environ $90 \%$ de la population sait lire et écrire. La politique d'éducation doit construire « la résistance et le socialisme ». S'inspirant des modèles chinois et soviétique, les programmes éliminent les traces de «l'enseignement réactionnaire» colonial et s'orientent vers l'enseignement du marxisme léninisme qui est toujours d'actualité. Mais trente années de guerre postcoloniale, française puis américaine, de 1945 à 1975, laissent le pays ravagé et ce premier système éducatif exsangue.

5 Après la réunification du pays en 1975, la troisième réforme éducative de 1979 établit un système national unifié d'éducation en accord avec les principes d'une éducation gratuite pour tous ${ }^{4}$ et en intégrant les principes de l'idéologie socialiste. L'unification du système est achevée en 1993 sur une base de douze années (5-4-3).

6 Le système actuel découle de ces changements et de l'adaptation à la politique du renouveau, qui officialise, à partir de 1986, le passage d'une économie planifiée à une économie orientée par le marché, ainsi qu'à la politique de la socialisation adoptée en 1996. Cette dernière revient à demander à tous les acteurs, dont les familles et les communautés locales, de participer au financement de l'éducation. La socialisation a une double finalité : elle vise d'une part un élan unificateur autour de l'objectif de l'éducation du et par le peuple pour le développement du pays, elle légitime d'autre part la marchandisation du secteur avec l'ouverture d'établissements privés et d'écoles du peuple financées localement, compte tenu de l'insuffisance des moyens.

\section{L'enseignement général jusqu'au secondaire}

7 Dans la loi pour l'éducation de 2005, le système éducatif vietnamien se compose du préscolaire, du primaire, des deux cycles du secondaire et du supérieur. À ces différents niveaux, la distinction est faite entre la filière générale et les filières techniques et professionnelles, l'enseignement non formel et la formation continue.

Il existe aujourd'hui trois types d'établissements au Viêt Nam : les écoles publiques, les écoles du peuple et les écoles privées. Les écoles du peuple sont des établissements à but non lucratif, créés à l'initiative de personnes ou d'organisations, et dont le financement est assuré par les autorités locales, par des dons et par les frais de scolarité payés par les familles. Les écoles privées sont, elles, financées par les frais de scolarité. Elles peuvent dégager des profits et paient à ce titre taxes et impôts. Cette évolution s'est accompagnée d'une dégradation de l'enseignement, la richesse devenant le principal déterminant de la scolarisation.

\section{Le préscolaire}

9 Le préscolaire comporte les crèches et garderies familiales pour les enfants âgés de 3 à 36 mois, et les écoles maternelles pour les enfants âgés de 3 à 6 ans. Conséquence d'une offre publique faible et de la politique de socialisation de l'éducation, plus de $61 \%$ des écoles du préscolaire sont aujourd'hui privées. Les taux brut de scolarisation étaient de $39 \%$ en 1999 et de $60 \%$ en 2005, mais les inégalités sont grandes entre les régions et les 
niveaux de vie. Les familles perçoivent mal l'intérêt de cette scolarisation précoce, alors qu'elles ont depuis longtemps assimilé l'importance de l'éducation pour l'avenir de leurs enfants et comptent sur le soutien que ces derniers leur apporteront dans leurs vieux jours. Aussi, la scolarisation précoce se développe lentement dans les milieux ruraux.

\section{Le primaire}

Jusqu'à récemment, seules les écoles publiques avaient le droit de proposer une scolarisation à ce niveau, le primaire étant en effet gratuit et obligatoire. L'école primaire comporte cinq niveaux (classes 1 à 5) et accueille les enfants âgés de 6 à 10 ans. Compte tenu du manque d'écoles, de classes et de la pénurie d'enseignants, il existe encore de nombreuses classes à double flux, soit le matin, soit l'après-midi. À quelques rares exceptions près, chaque commune dispose au moins d'une école primaire.

11 L'une des principales préoccupations tient à l'abandon des études en cours ou à la fin de ce cycle, à quoi s'ajoute un faible niveau des acquis des élèves. Le taux net de scolarisation est passé de $95 \%$ en 1999 à $86 \%$ en 2005, selon le rapport de l'éducation pour tous (Unesco, 2009). Plus d'un million d'enfants âgés de 6 à 10 ans étaient non scolarisés en 2005, contre 447000 en 1999. Endiguer les sorties précoces et l'abandon de la scolarité est l'un des défis actuels du pays, tant au niveau du primaire qu'aux niveaux suivants.

\section{Enseignement secondaire de base}

12 L'enseignement secondaire de base regroupe les classes 6 à 9 et accueille les enfants âgés de 11 à 14 ans. La loi sur l'éducation de 2005 définit la généralisation de l'éducation de base comme une priorité. Au 31 décembre 2007, le Vietnam scolarisait 5,8 millions d'enfants dans environ dix mille établissements ${ }^{5}$ pour le premier cycle de l'enseignement secondaire général. Le taux brut de scolarisation était de $88 \%$ en $2005^{6}$. Comme pour le primaire, les abandons sont fréquents à ce niveau.

Dans certaines régions industrielles ou dans les zones où l'agriculture constitue l'essentiel des débouchés, l'utilité du secondaire supérieur apparaît faible aux familles, rapportée aux coûts induits (frais de scolarité, manque à gagner du fait de l'inactivité professionnelle des jeunes, coûts de transport voire d'hébergement, etc.). Malgré les efforts de généralisation de l'éducation de base universelle et la lutte contre l'abandon scolaire, en particulier à travers les classes de généralisation ${ }^{7}$, le choix est vite fait par les enfants des familles les plus pauvres qui arrêtent leur scolarité pour partir travailler.

\section{Enseignement secondaire supérieur}

14 Le deuxième cycle d'enseignement secondaire (classes 10 à 12) rassemble les élèves âgés de 15 à 18 ans. Les effectifs ont été multipliés par trois entre 1995 et 2007 avec 3,02 millions d'élèves dans plus de 2100 établissements. Néanmoins, le taux brut de scolarisation reste très faible à $59 \%$ en 2005 , avec une tendance à y envoyer de préférence les garçons, le taux de scolarisation féminin étant à $46 \%$ (dans 
l'enseignement supérieur, il est de $1 \%$ en 1993 ; le taux d'analphabétisme féminin atteint au total 9,8\%).

15 La réussite à l'examen de fin de cycle donne le baccalauréat (Trung ho.c phổ thông) et permet de passer les examens d'entrée à l'université. Si elle est appliquée dès 2009-2010, l'une des mesures de la réforme en cours devrait tenter de fusionner l'examen final avec celui d'entrée à l'université.

Les examens de fin de secondaire et ceux d'entrée à l'université ont une très grande importance dans la vie des élèves du secondaire et de leurs parents. Offrant la possibilité d'accès à l'université, leur réussite est une condition incontournable pour un avenir meilleur pour la famille. Cette nécessité de réussir, associée au mode d'évaluation des enseignants sur la base de la réussite de leurs élèves aux examens, s'est transformée en obsession, source de tricheries et de corruption contre lesquelles les autorités s'activent. Hô Chi Minh en son temps demandait déjà, au nom des vertus morales du confucianisme, d'extirper la prévarication, la bureaucratie et le gaspillage... ces maux sont constants.

17 La faiblesse des acquis des élèves découle pour partie d'un mode d'enseignement centré sur la diffusion de savoirs qui mobilisent surtout l'apprentissage par cœur au détriment de la réflexion et de la participation active des élèves. L'héritage confucéen peut expliquer une partie de ces attitudes. D'autres explications mettent en avant un trop grand nombre d'élèves par classe, ainsi que la lourdeur et l'inadaptation de programmes suivis à la lettre, quel que soit le degré d'assimilation des élèves.

\section{L'enseignement technique et professionnel}

18 En 2007, l'enseignement secondaire professionnel comprenait presque 300 établissements regroupant 710000 élèves. L'enseignement technique et professionnel commence essentiellement à l'issue du secondaire de base ou du secondaire supérieur.

19 Les écoles professionnelles d'enseignement secondaire dispensent des formations de trois à quatre années, selon les disciplines, et associent des enseignements spécialisés à des enseignements généraux.

Les établissements d'enseignement technique prennent en charge la formation des élèves, soit à l'issue du collège pour un programme de trois ans à quatre ans, soit après le lycée pour un programme entre un et trois ans.

21 La réussite aux examens de fin du secondaire technique ou professionnel donne le droit d'accès, après examen d'entrée, à l'université ou dans des cycles courts supérieurs, à travers des écoles normales supérieures, des écoles polytechniques, etc. La multiplicité des tutelles de cet enseignement technique et professionnel (ministères, provinces, chambres de commerce et de l'industrie, établissements privés/public) ne facilite pas un pilotage cohérent et efficace. Néanmoins, ces tutelles cherchent toutes à développer un financement mixte public/privé.

22 À l'instar de beaucoup de pays, l'enseignement technique et professionnel est le parent pauvre de l'éducation au Viêt Nam, alors qu'il permettrait à plus d'élèves de continuer leur scolarité tout en répondant aux besoins du pays en main-d'œuvre technique qualifiée aux niveaux secondaire et supérieur. 


\section{Le supérieur}

23 Les effectifs ont doublé et sont passés à plus de 1928000 en 2008, du fait de l'évolution de la structure démographique, d'une plus grande ouverture de l'université, de l'augmentation du niveau de vie moyen et de la demande d'éducation croissante. Entre 1999 et 2005, les taux brut de scolarisation ont gagné cinq points de pourcentage pour atteindre $16 \%$. Mais ceux de la zone Asie de l'Est eux ont presque doublé à $24 \%$.

Le supérieur comprend d'une part, une multitude de formations courtes de deux ou trois ans qui sont proposées par certaines universités, ainsi que par des écoles normales supérieures ou polytechniques. Par ailleurs, l'université représente environ $57 \%$ des établissements d'enseignement supérieur et offre des formations de trois à quatre années ${ }^{8}$ qui préparent aux diplômes d'études universitaires. À l'issue de l'examen, les étudiants peuvent poursuivre dans le post-universitaire : master (deux ans) et doctorat (trois à quatre ans).

Face au manque d'universités, d'enseignants, aux coûts de construction, d'entretien et de fonctionnement, les ministres successifs ont favorisé les partenariats avec l'étranger et le développement d'une offre privée. Fin 2008, sur les 369 universités et écoles supérieures du pays, environ $17 \%$ étaient privées. Aujourd'hui, alors que $13,7 \%$ des étudiants sont dans le privé, le ministère en attend $30 \%$. Plus étonnant encore dans une république socialiste, un projet récent visait à transformer les universités en sociétés par actions... Les oppositions furent vives.

Le défi de l'enseignement supérieur est triple : trouver des financements suffisants eu égard aux besoins, mettre en œuvre une gestion adéquate pour développer une offre de formation de qualité (dont celle des enseignants), ne pas aggraver les inégalités d'accès.

\section{Gouvernance, financement et pilotage du système éducatif}

27 Les services d'éducation ont été unifiés sous l'égide du ministère de l'Éducation et de formation (MEF) en 1993. Après celle de 1998, c'est la loi du 14 juin 2005 qui a cours aujourd'hui. Ses orientations confortent un double mouvement de décentralisation et de marchandisation.

\section{Structure et organisation}

Le plan de stratégie éducative pour $2009-2020^{9}$ conforte cette orientation. Le MEF coordonne le financement et la gestion du système éducatif au niveau central. Il s'appuie sur les services de l'éducation et de la formation et sur les comités populaires locaux $^{10}$ aux niveaux des provinces, districts et communes, ce qui impose aux établissements scolaires une sorte de double tutelle peu commode et peu efficace.

Le ministère définit le contenu des programmes et les méthodes d'enseignement à tous les niveaux. Il s'occupe aussi de la publication des manuels scolaires, des cahiers d'exercices et des livres des professeurs à travers la maison d'édition de l'éducation (Nhà xuâ't bản Giáo du.c). Il définit l'organisation des cycles d'enseignement, des examens, de la certification et des diplômes, les critères d'affectation des moyens en infra-structure et en personnel enseignant. Qu'il s'agisse des établissements publics, 
populaires voire privés, le ministère est censé réaliser une gestion unifiée de la formation, du développement de carrière et de l'affectation des enseignants. À cette fin, il s'appuie en partie sur un contrôle réalisé par un corps d'inspection mais aussi et surtout sur les services déconcentrés du ministère au niveau des provinces (64 départements de l'éducation et de la formation) et des districts (plus de 500 bureaux de l'éducation et de la formation). Ces services, ainsi que les comités populaires des différents niveaux, relaient la politique générale d'éducation dans les provinces en fonction des contextes et des budgets affectés (une partie des financements vient de l'État central et une autre de sources locales comme les frais de scolarité).

Selon la loi de 2005, l'enseignement supérieur général, technique et professionnel, ainsi que les programmes nationaux relèvent de l'État central. Le secondaire supérieur est sous l'autorité des services de l'éducation et de la formation de la province tandis que le pré-scolaire, le primaire et l'enseignement secondaire de base dépendent des comités populaires et des services de l'éducation et de la formation du district.

\section{Financement et décentralisation}

31 En 2007, la dépense publique totale en éducation était de 66770 milliards de $d^{-}{ }^{-}{ }^{\prime} n g s^{11}$ (2,8 milliards d'euros) dont $17,2 \%$ en dépenses d'infrastructures, $82,7 \%$ pour les dépenses courantes (la plus grande partie de ces ressources est affectée au traitement des enseignants), et $5,1 \%$ pour le programme en faveur des minorités ethniques ${ }^{12}$. Le 15 mai 2009, lors de la deuxième conférence des ministres de l'éducation d'Asie-Europe, le premier ministre vietnamien, a déclaré que les dépenses d'éducation de son pays atteignaient $20 \%$ du budget de l'État (contre 9,7\% en 1991 et $15 \%$ à la fin des années 90). Avec $25 \%$, l'éducation est la part la plus importante du plan prévisionnel de dépenses publiques pour 2009. En revanche, conséquence de la décentralisation et des autres priorités politiques, le budget de l'éducation et de la formation ne représente plus que 9,2\% des dépenses de l'État central. L'État dépense $80000 d^{-} \hat{o}$ 'ngs pour un élève dans le primaire, 10000 dans le secondaire $1^{\mathrm{er}}$ cycle, 170000 dans le $2^{\mathrm{e}}$ cycle, 2470000 dans le supérieur. La Banque mondiale ${ }^{13}$ fait remarquer que chaque étudiant de l'enseignement supérieur, généralement issu d'une famille riche, coûte trente fois plus qu'un élève du primaire.

32 Le budget global pour l'éducation et la formation est constitué de fonds publics et de fonds non étatiques. Les premiers correspondent au budget de l'État pour l'éducation, à l'aide publique au développement et aux prêts. Les fonds non étatiques sont constitués des frais de scolarité, des frais payés par les familles, des financements par les entreprises, des dons, ...

Le MEF contrôle le budget global et en ventile une partie aux autorités locales, selon leurs responsabilités respectives. À la suite de la socialisation de l'éducation et de la décentralisation, une bonne part des financements provient de sources locales, et donc des familles ${ }^{14}$ dont la charge s'est alourdie.

\section{Défis et enjeux actuels}

Les défis et enjeux sont nombreux. Il faut d'une part consolider les objectifs de l'éducation universelle pour tous, c'est-à-dire endiguer l'abandon scolaire et assurer un accès à la scolarité réel et équitable surtout pour les minorités ethniques ${ }^{15}$ et les 
populations rurales. Malgré de nouveaux mécanismes de péréquation entre les provinces, les régions les plus pauvres disposent des budgets les plus faibles. Or c'est dans ces régions où les ethnies sont les plus présentes que les taux de scolarisation sont les plus faibles et que l'abandon est le plus fréquent.

D'autre part, face aux besoins de financement du secteur éducatif, le gouvernement favorise le développement d'une offre privée, alors que la qualité des sortants, en particulier dans le supérieur, est loin d'être garantie. Pourtant, pour assurer sa croissance, le pays a un besoin urgent de main-d'œuvre qualifiée. Les entreprises étrangères installées dans le pays peinent à trouver des cadres vietnamiens opérationnels.

Enfin, compte tenu de la faiblesse des acquis des élèves à tous les niveaux, les curricula et les méthodes d'enseignements sont appelés à évoluer vers plus d'application des savoirs et par l'élaboration de cursus qui valorisent l'esprit critique, la réflexion et la prise d'initiative. Cela passe par une augmentation du nombre d'enseignants, par une élévation de leur qualification et de leur rémunération afin qu'ils puissent se concentrer sur leurs classes d'enseignement et non sur des activités annexes ou la dispense de cours supplémentaires plus lucratifs.

Le système éducatif vietnamien, pour partie héritier de la logique confucéenne, est à un moment clé de son évolution. Confronté aux réalités économiques, sociales et culturelles de l'ouverture internationale, il doit opérer sa transformation, alors qu'il est tiraillé entre les cadres contraignants d'une république socialiste et une modernisation nécessaire qu'il aborde, entre autres, par la décentralisation et la marchandisation, pas toujours maitrisées, de l'éducation.

Quelques données

\begin{tabular}{|c|c|}
\hline \multicolumn{2}{|c|}{ Superficie : $331221 \mathrm{~km}^{2}$} \\
\hline \multicolumn{2}{|c|}{ Devise : «Indépendance, liberté, bonheur» } \\
\hline \multicolumn{2}{|c|}{$\begin{array}{l}\text { Organisation administrative: } \\
64 \text { provinces ou villes-provinces, } \\
\text { partagées en districts }\end{array}$} \\
\hline \multicolumn{2}{|c|}{ Population : 87 millions } \\
\hline \multicolumn{2}{|c|}{ PIB par habitant en $2007^{\star}: 770$ dollars } \\
\hline $\begin{array}{l}\text { Structure de la } \\
\text { - Primaire } \\
\text { - Secondaire } \\
\text { - Tertiaire }\end{array}$ & $\begin{array}{l}\text { population active : } \\
22,10 \% \\
39,73 \% \\
38,17 \%\end{array}$ \\
\hline \multicolumn{2}{|c|}{ Espérance de vie à la naissance : 74 ans } \\
\hline $\begin{array}{l}\text { Taux d'alphabe } \\
\left(105^{\mathrm{e}} \text { place sur }\right.\end{array}$ & $\begin{array}{l}\text { étisation des adultes : } 90 \% \\
177 \text { ) }\end{array}$ \\
\hline
\end{tabular}

Source : Banque mondiale 


\section{BIBLIOGRAPHIE}

Recherche sur la qualité de l'éducation dans la province de Khán Hòa, Viêt Nam, Nolwen Henaff et Trần Thi. Thuâ.n (dir.), Aide et Action, 2008.

Viet Nam's Education in Transitional Period, Nguyễn Lô.c, National Institute of Education Strategy and Curriculum, Ministère de l'éducation et de la formation, 2006.

Education Law, National Assembly of the socialist republic of Vietnam, loi 38/2005/QH11, 2005.

Voyage dans les cultures du Viêt Nam, Lê Thành Khôi, Horizons du Monde, 2001.

Education et Civilisations, Thành Khôi, UNESCO, Horizons du Monde, 2001.

Population et développement au Viêt Nam, Patrick Gubry (dir.), Karthala-CEPED, 2000.

\section{NOTES}

1. L'auteur remercie Pierre-Louis Gauthier pour les éclairages historiques qu'il a bien voulu lui apporter.

2. Trinh Van Thao, Viêt Nam du confucianisme au communisme, Paris, L'Harmattan, 1990.

3. Cette écriture romanisée a été développée par le jésuite Alexandre de Rhodes en 1651.

4. La Constitution de 1967 garantit l'accès à l'éducation à tous, l'éducation de base est gratuite et obligatoire.

5. Toutes les communes n'ont pas encore un établissement secondaire propre.

6. Rapport de l'Unesco sur l'éducation pour tous (EPT), 2008.

7. Dispositif de rétention et d'aide au retour à l'école pour les enfants qui ont abandonné prématurément leurs études. Ces classes spécifiques regroupent des enfants de 6 à 14 ans. Les minorités ethniques y sont surreprésentées.

8. Six années pour la médecine.

9. Une réforme générale du système pour 2009-2020 a été soumise à l'Assemblée nationale fin mai 2009. Une réforme des mécanismes de financement de l'éducation pour 2009-2014 est en discussion.

10. Au niveau local des provinces, districts et communes, les comités populaires assurent le pouvoir exécutif.

11. Source : MEF.

12. Le Vietnam comporte 54 ethnies différentes.

13. Banque mondiale, Viet Nam : Poverty Assesment and Strategy, 1995.

14. L'Assemblée nationale considère que la part des ménages part ne devait pas être supérieure à $5 \%$ des frais de scolarité (années 1 à 12 ).

15. Certaines ethnies du nord et des hauts plateaux du centre cumulent les handicaps : faible scolarisation, fort abandon. 
INDEX

Mots-clés : école, enseignement, sciences de l'éducation

Palabras claves : escuela, enseñanza, ciencias de la educación

Keywords : school, teaching, educational sciences

Index géographique : Vietnam

AUTEUR

SÉBASTIEN ASSERAF-GODRIE

Sébastien Asseraf-Godrie est agrégé de sciences sociales et Inspecteur d'académie-Inspecteur pédagogique régional en sciences économiques et sociales. Il a vécu au Vietnam. 